\title{
Bruno RESTIF, La Révolution des paroisses. Culture paroissiale et Réforme catholique en Haute-Bretagne aux $\mathrm{XVI}^{e}$ et XVII ${ }^{e}$ siècles
}

\section{Bernard Merdrignac}

\section{(2) OpenEdition Journals}

\section{Édition électronique}

URL : https://journals.openedition.org/abpo/572

DOI : $10.4000 / a b p o .572$

ISBN : 978-2-7535-1504-8

ISSN : 2108-6443

\section{Éditeur}

Presses universitaires de Rennes

\section{Édition imprimée}

Date de publication : 30 décembre 2006

Pagination : 206-210

ISBN : 978-2-7535-0405-9

ISSN : 0399-0826

\section{Référence électronique}

Bernard Merdrignac, "Bruno RESTIF, La Révolution des paroisses. Culture paroissiale et Réforme catholique en Haute-Bretagne aux xvI et xvII' siècles », Annales de Bretagne et des Pays de l'Ouest [En ligne], 113-4 | 2006, mis en ligne le 30 décembre 2008, consulté le 22 juillet 2022. URL : http:// journals.openedition.org/abpo/572; DOI : https://doi.org/10.4000/abpo.572

Ce document a été généré automatiquement le 22 juillet 2022.

Tous droits réservés 


\title{
Bruno ReSTIF, La Révolution des paroisses. Culture paroissiale et Réforme catholique en Haute-Bretagne aux XVI et $\mathrm{XVII}^{e}$ siècles
}

\author{
Bernard Merdrignac
}

\section{RÉFÉRENCE}

Rennes, PUR/SHAB, 2006, 420 p.

\section{NOTE DE L'ÉDITEUR}

Cet ouvrage sur le site des Presses universitaires de Rennes : notice, documents et commande en ligne : http://www.pur-editions.fr/detail.php?idOuv=1043

1 Ce livre est la version allégée et remaniée de la thèse de doctorat intitulée « La paroisse, cadre d'application de la Réforme catholique en Haute-Bretagne (diocèses de Rennes, Dol et Saint-Malo) - Histoire d'un processus de transformation religieuse et culturelle $\left(\mathrm{XVI}^{\mathrm{e}}\right.$ et $\mathrm{XVII}^{\mathrm{e}}$ siècles) » soutenue par l'auteur en décembre 2004. Le cliché qui illustre la couverture de l'ouvrage exprime d'emblée le propos de Bruno Restif qui s'est attaché à dégager sur le long terme ( $\mathrm{du}$ milieu $\mathrm{du} \mathrm{Xv}^{\mathrm{e}}$ siècle au milieu du XVIII ${ }^{\mathrm{e}}$ siècle) les interactions entre la "Réforme catholique » et le cadre paroissial préétabli dans lequel celle-ci s'est inscrite. Dans l'église de Tréfumel, modeste paroisse du diocèse de SaintMalo, un retable et un balustre ont certes remplacé la maîtresse vitre et le chancel afin de rendre visible la célébration eucharistique. Sur le maître-autel, le tabernacle (innovation $d u \mathrm{XVII}^{\mathrm{e}}$ siècle destinée à magnifier la présence réelle) s'emboîte dans le retable comme une réduction en miniature de celui-ci. Mais ici, à la différence de la 
plupart des édifices comparables, les réaménagements demeurés partiels font ressortir la dialectique entre souci d'unification et volonté de délimitation de l'espace religieux. Le chœur roman est séparé de la nef par un des rares arcs triomphaux conservé en Haute Bretagne. La poutre de gloire est restée en place et des autels latéraux (surmontés ultérieurement de petits retables) subsistent contre les murs de l'arc diaphragme. Ainsi la séparation entre clercs et laïques qui s'est instaurée à la fin du Moyen Âge prélude au renforcement de la distinction entre sacré et profane qui constitue l'un des enjeux de la Réforme. Dès lors, celle-ci. apparaît donc moins comme une rupture avec l'époque antérieure que comme l'aboutissement d'un processus amorcé dès le milieu du XIV siècle.

2 Si l'on a jugé bon d'ouvrir ce compte rendu en mettant l'accent sur la relation entre les évolutions artistiques et les applications liturgiques de la Réforme catholique, c'est que l'étude de B. Restif constitue un front d'avancée de la recherche sur l'" art paroissial », restée relativement en friches pour le pays gallo alors même qu'elle bénéficiait de nombreux travaux concernant la Basse Bretagne. L'auteur a pris le parti de croiser systématiquement l'analyse des monuments, des objets liturgiques ou de la statuaire avec la critique des sources imprimées (ouvrages liturgiques, par exemple) et le dépouillement des sources manuscrites : registres paroissiaux, archives de confréries, testaments et surtout comptes de fabriques (déposés dans les archives publiques depuis les années 1990). Les lecteurs des Annales de Bretagne et des pays de l'Ouest ont d'ailleurs pu apprécier tout l'intérêt de cette démarche dans deux articles de B. Restif récemment publiés par la revue ( Pouvoir et religion dans une paroisse rurale de Haute-Bretagne au XVI ${ }^{\text {e }}$ siècle : le compte de fabrique de Louvigné-de-Bais (1503-1504)» [en coll. avec Y. Lagadec], t. 109 (2002) no 1- «Réforme catholique et modifications de l'espace sacré. Architecture et organisation de l'espace intérieur des édifices cultuels », t. 110 [2003], $\mathrm{n}^{\circ} 4$ ). Depuis une vingtaine d'années, alors que la partie occidentale de la Bretagne faisait l'objet de recherches magistrales en histoire religieuse de la part des modernistes (A. Croix, G. Provost), la Haute Bretagne se voyait réserver la portion congrue et ne faisait fonction, la plupart de temps, que de terme de comparaison. C'est pourquoi, B. Restif a délibérément limité son terrain d'enquête aux diocèses de Rennes, Dol et Saint-Malo afin d'y analyser la vie religieuse de 436 paroisses (et 33 trèves) en prenant en compte les villes petites et moyennes (mais excluant les cités épiscopales) avec l'ambition de faire émerger des phénomènes jusqu'ici passés inaperçus. Il s'agit donc d'une thèse, dans tous les sens du terme, qui s'articule selon une progression logique rythmée par des conclusions partielles qui récapitulent vigoureusement chaque étape de l'argumentation. En conclusion générale, l'auteur revendique la «fonction sociale de la recherche historique » qui se veut «intelligibilité du passé qui soit aussi intelligibilité du présent ». Mais il n'est pas inavouable non plus de la part du signataire du présent compte rendu d'avoir gratuitement pris plaisir à suivre les avancées d'une recherche rigoureuse exposée avec élégance et clarté.

3 En effet, l'érudition de B. Restif est rarement prise en défaut et il serait mal venu de faire grief à celui-ci de quelques inexactitudes en ce qui concerne la période médiévale. Il convient plutôt de lui savoir gré de souligner que la Réforme catholique conçue, dès la fin du XIV siècle, comme une "nouvelle christianisation", a été menée dans un réseau paroissial contemporain de la "première christianisation ", à la charnière de l'Antiquité tardive et du haut Moyen Âge. La part prise par les « moines venus d'outreManche » dans l'évangélisation du diocèse d'Alet (devenu celui de Saint-Malo au 
XII ${ }^{e}$ siècle) valait donc d'être rappelée ici. Mais il est inexact de préciser qu'une partie d'entre eux «n'appartient pas à l'origine aux populations qui s'installent alors en Armorique ». La filière irlandaise invoquée en note relève du mythe hagiographique et le cas de saint Colomban n'est que l'exception qui confirme la règle. Certes, l'éponyme de Saint-Coulomb a peut-être débarqué ici, mais rien ne prouve que le séjour de saint Malo à Luxeuil selon sa Vita médiévale ait une réalité historique et les frères installés « in viciniam Britonnum » à qui Colomban fait référence dans sa correspondance (Ep. IV) l'étaient plutôt en Nantais qu'à proximité d'Alet. Dès lors, une certaine confusion s'instaure entre "saints locaux, martyrs et autres saints du Moyen Âge " (voire "non identifiés" - annexes XIII et XIV consacrées à la statuaire) et celle-ci a des répercussions sur les précieux développements consacrés par B. Restif au culte des saints. La mention en 1574 dans les comptes de fabrique de Gévézé (diocèse de Rennes) des « ymaiges de st Men, st Ugnac, st Day, st Cymphorien et st Gobryain » n'amalgame pas des "saints de diverses origines » comme le pense l'auteur. En effet, saint Symphorien n'est probablement pas le martyr d'Autun du III $^{\mathrm{e}}$ siècle, mais plutôt le saint celtique Kiferian qui a été souvent été confondu avec lui. Les quatre autres statues sont bien celles de saints bretons. Outre saint Méen dont la paroisse qui lui doit son nom dans le diocèse de Saint-Malo faisait l'objet d'un célèbre pèlerinage à l'échelle du royaume, on peut identifier saint Gobrien, évêque de Vannes, vénéré dans les missels rennais de1492 et de 1588. Saint « Ungac » éponyme de l'enclave doloise de Saint-Uniac dans le diocèse de Rennes doit être identifié au saint breton Winniau qui fait l'objet de nombreuses études spécialisées. Quant à saint « Day » (qui a sans doute posé problème à l'auteur puisqu'il insère des [...] à la place de son nom dans la citation de la p. 85), on reconnaît en lui l'énigmatique saint Dei (ou They, devenu parfois Sainte-Ayde) qu'il est intéressant de trouver orthographié sous la forme St-Day qu'il revêt dans la toponymie du Cornwall. C'est pourquoi, il est d'autant plus significatif de voir ces saints disparaître dans les sources $d u X$ VII $^{e}$ siècle qu'il ne s'agit pas simplement de saints "vénérés à l'échelle locale », mais d'un groupe de saints celtiques qui vaudrait d'être considéré à ce titre comme significatif.

Certaines approximations auraient sans doute gagné à être rectifiées dans cette version imprimée de la thèse. C'est peut-être par étourderie (à moins qu'il s'agisse d'une simple coquille) que l'auteur date de la fin du $\mathrm{xI}^{\mathrm{e}}$ siècle l'abandon par l'évêché de Dol de ses prétentions métropolitaines, alors que la rivalité avec Tours a traîné en longueur durant tout le XII ${ }^{\mathrm{e}}$ siècle jusqu'à ce qu'Innocent III tranche la querelle en 1199. De ce fait, le synchronisme apparent qui ferait « aussi » du xI siècle « l'époque de la création des archidiaconés et des doyennés » tombe de lui-même. La carte [3] et l'annexe [1] qui présentent ces circonscriptions auraient d'ailleurs sans doute mérité un commentaire plus développé puisque leur mise en place fait l'objet de débats en cours. M. Brand'honneur dans sa récente étude des Manoirs et châteaux du comté de Rennes (PUR, 2001) a émis l'hypothèse séduisante que, dès l'époque carolingienne, le doyenné correspondait à la vicaria ou pagus minor. Longtemps négligées par les historiens, ces institutions intermédiaires entre la paroisse et le diocèse, contribuent pourtant à l'encadrement religieux des campagnes dans le parti pris par l'auteur d'identifier « au raz du sol » les acteurs du changement plutôt que de partir du sommet de la hiérarchie. Ce renversement de perspective n'empêche pas B. Restif de se monter attentif aux impulsions épiscopales qui préludent à la Réforme catholique. En présentant le personnel « plus politique » (selon l'expression de G. Bedouelle) qui accède à l'épiscopat au cours $\mathrm{du} \mathrm{XVI}^{\mathrm{e}}$ siècle, l'auteur affirme, un peu trop rapidement sans doute, que 
François $\mathrm{I}^{\mathrm{er}}$ voulut faire appliquer dans la nouvelle province le Concordat de Bologne signé avec le pape Léon X (1516) qui assurait au roi sinon «le choix des évêques », du moins une grande latitude dans les nominations épiscopales. Au contraire, dans leur récente mise au point sur l'Union de la Bretagne à la France (Skol Vreizh, 2003), D. Le Page et M. Nassiet rappellent que le duché fut prudemment excepté du concordat, demeurant "pays d'obédience ", ce qui n'empêchait pas bien entendu François Ier de saisir toutes les occasions pour accroître sa main-mise sur l'administration de la Bretagne, confiant, par exemple, la charge de vice-chancelier à Jean Briçonnet (1514). Cette grande famille de la bourgeoisie administrative tourangelle donne trois évêques successifs pendant plus d'un demi-siècle à Saint-Malo puisque Guillaume Briçonnet (1493-1513) cède sa charge à son fils Denis (1513-1535) qui lui-même la transmet à son neveu François Bohier (1535-1569). Lié par son frère aîné Guillaume au "groupe de Meaux » autour de Lefèvre d'Étaples, Denis Briçonnet, tout en cumulant les bénéfices et en ne perdant pas de vue les intérêts familiaux, se préoccupe du niveau spirituel de son clergé à l'intention duquel il fait imprimer à Nantes en 1518 l'Instruction des Curez pour instruire le simple peuple dont un des deux seuls exemplaires subsistant est conservé à la médiathèque de Dinan. L'analyse de ce manuel (dans lequel l'influence de Jean Gerson est nettement perceptible) donne à B. Restif l'occasion de quelques-unes des meilleurs pages de son livre. Par contre, il n'y a pas lieu, comme il le fait, de noter le "décalage » de Denis Briçonnet par rapport aux diocèses voisins en ce qui concerne l'impression de bréviaires à l'usage du clergé. Cela serait d'autant plus étonnant que son frère aîné Guillaume a fait de son côté imprimer des bréviaires pour les diocèses de Lodève, puis de Meaux lorsqu'il en assumait l'épiscopat. L'affirmation de l'auteur selon laquelle «le premier Bréviaire imprimé recensé pour le diocèse de Saint-Malo date de 1603 " s'appuie sur la Bibliographie der Breviere, 1501-1850 d'Hans Bohatta. Ce catalogue qui rend d'incontestables services doit cependant être vérifié - ne serait-ce que parce qu'il lui arrive de confondre Dôle et Dol! En fait, jusqu'aux bombardements d'août 1944, la Bibliothèque municipale de Saint-Malo abritait un bréviaire sans doute imprimé durant l'épiscopat de Denis Briçonnet. En effet, les Bollandistes qui en ont extrait des leçons pour les Acta Sanctorum l'ont daté de 1517 (c'est-à-dire qu'il serait pratiquement contemporain de l'Instruction des Curez, à moins de retenir la date de 1537 donnée par $\mathrm{F}$. Duine). Au reste, dès 1489 , un bréviaire malouin (perdu) avait été imprimé par Jean Higman à Paris, donc avant les bréviaires répertoriés ici par l'auteur pour Rennes (1512, 1514, 1533) et Dol (1519 et v. 1520).

5 Bien entendu, ces remarques ponctuelles ne remettent pas en cause les thèses de $B$. Restif. Celui-ci prend soin, en note à l'issue de son «Introduction", de justifier le plan adopté. Le dynamisme du Xvi ${ }^{e}$ siècle (interrompu provisoirement par les troubles de la Ligue qui donnent lieu à d'excellents développements sur les limites de l'impact de la "Réformation " protestante à partir des travaux de J.- Y. Carluer) prélude au grand siècle de la mise en œuvre de la Réforme catholique dans les paroisses qui s'ouvre ici dans les années 1610 et se poursuit jusqu'au premier tiers du XvIII ${ }^{\mathrm{e}}$ siècle. Le passage d'une première partie consacrée aux prémices de l'entreprise réformatrice ( les paroisses de Haute Bretagne au $\mathrm{XVI}^{\mathrm{e}}$ siècle ») à une seconde partie exposant la mise en œuvre de celle-ci (« le siècle de la réforme catholique ») se donne certes à lire de façon chronologique. Mais il correspond en fait à un raisonnement logique débouchant sur une dernière partie consacrée aux « croyances et pratiques » qui évalue les « réussites et limites de la Réforme catholique ». Un des apports essentiels de l'ouvrage consiste à relativiser (du moins en ce qui concerne la Haute Bretagne) le schéma préconçu d'une 
diffusion du courant réformateur à partir des villes en direction des campagnes. L'auteur montre qu'il s'agit moins d'un décalage chronologique que de différences dans les modalités d'application. Un autre point fort du présent ouvrage est qu'il renouvelle en le précisant le portrait collectif de ce clergé paroissial (globalement bien connu pour la Haute Bretagne du XviII ${ }^{e}$ siècle ou la Basse Bretagne des années 1760-1840). On ne peut, au passage, que souscrire à l'insistance de B. Restif pour rectifier un contre-sens quant à l'attitude du célébrant durant la messe. Celui-ci ne tournait pas le dos aux fidèles (depuis l'époque carolingienne) comme on le lit trop souvent, mais prenait la tête d'une "assemblée en marche vers la Jérusalem céleste ». Le passage de " ministre des sacrements " médiéval (selon la définition de C. Vincent) au recteur moderne, qui s'accompagne d'une forte baisse des effectifs ecclésiastiques, s'effectue sur un siècle (comme l'a déjà montré A. Croix) : il porte ses premiers fruits au milieu du XVII ${ }^{\mathrm{e}}$ siècle et est acquis vers 1675. La promotion du « recteur» devenu un notable villageois est illustrée [cliché h.t. 17] par celui qui s'est fait représenter au début du XvIII ${ }^{e}$ siècle sur le mur extérieur de l'église de Saint-Gonlay (diocèse de Saint-Malo). Le volumineux registre manuscrit rédigé à partir de 1620 par dom Noël Georges (conservé aux archives diocésaines de Rennes et depuis peu en dépôt aux Archives départementales d'Ille-etVilaine) inédit mais bien connu des spécialistes et que B. Restif exploite à plusieurs reprises, aurait fourni une figure exemplaire du prêtre de la Réforme catholique. L'auteur, desservant de chapellenie à Maxent. (diocèse de Saint-Malo) qui a été l'élève de Pierre Porcher, prieur-recteur de la paroisse, ne tarit pas d'éloges envers son maître (résidence, construction de l'ossuaire, impression de l'office de saint Maxent, défense des revenus de son prieuré-rectorat). À l'instigation de l'évêque réformateur de SaintMalo Guillaume le Gouverneur (1610-1630) ce recteur s'efforce de promouvoir la «grande confrairie » du Saint-Sacrement qui semble déjà à Noël Georges «obscurcir le lustre» des anciennes confréries d'intercession dédiées à saint Maxent et saint Jean Baptiste. L'essor des confréries de dévotion, notamment de celle du Saint-Sacrement, est un indicateur de l'impact de la Réforme en milieu rural. Celles-ci ne concurrencent pas la structure d'encadrement paroissial, mais en constituent un complément efficace dans ce projet de (re)christianisation de la société.

6 La finalité de l'ouvrage explicitement revendiquée par B. Restif est de rendre intelligible la mutation culturelle majeure que représente aujourd'hui la fin de la « civilisation paroissiale » qui a perduré ici jusqu'aux années soixante du $\mathrm{xx}^{\mathrm{e}}$ siècle. Cet objectif est brillamment atteint ! La rupture brutale de l'adéquation entre la paroisse et la commune dans l'actuel diocèse de Rennes, Dol et Saint-Malo, a consisté en une restructuration du dispositif pastoral depuis 2003 et en une extension considérable des territoires paroissiaux. Cette recomposition des circonscriptions ecclésiastiques, inachevée et difficilement lisible de par son ampleur, est susceptible de se prêter à des recherches interdisciplinaires sur les notions de territoire, et plus largement d'organisation de l'espace dont il faut souhaiter le développement. 